\title{
The Branching Rate of the Random Olami-Feder-Christensen Model with a Generic Coordination Number
}

\author{
S. T. R. Pinho \\ Instituto de Física, Universidade Federal da Bahia, 40.210-340, Salvador, Bahia, Brazil \\ and C. P. C. Prado \\ Instituto de Física, Universidade de São Paulo, Caixa Postal 66318, 05315-970, São Paulo, SP, Brazil
}

Received on 9 April, 2003

\begin{abstract}
In this paper we review and discuss some fundamental aspects of the random version of the Olami-FederChristensen model, and its relevance for the understanding of self-organized criticality (SOC). We review the universal character of the exponent $\tau=3 / 2$, related to avalanche size distributions in random SOC models, and its connection to branching processes theory. We also generalize previous results, that had been obtained for the random OFC model with four neighbors, to any coordination number. Finally we present some connections between our generalization and recent discussions involving the branching rate approach to this model.
\end{abstract}

\section{Introduction}

The concept of self-organized criticality was introduced by Bak, Tang and Wiesenfeld [1] in 1987, as a possible explanation of scale invariance in nature. To illustrate their basic ideas, they present a cellular automaton model that became known as the sandpile model, because of an analogy between its dynamical rules and the way sand topples and generates avalanches, in a real sand pile. Since this seminal work, a great number of cellular automata and coupled map models have been investigated, in an attempt to elucidate the essential mechanisms hidden in such a wide class of different non-linear phenomena whose statistics of events (or 'avalanches') are governed by power-laws. However, despite many efforts, up to now, one still lacks from a general theoretical framework for self-organized criticality. Most of the available results are purely numerical. Success in analytical investigations have been achieved mainly in the study of a special class of models that became known as abelian models [2], in mean-field type calculations [3-9] or through a renormalization group approach [10].

In this paper we discuss the random neighbor version of the Olami-Feder-Christensen (R-OFC) model. The original OFC model introduced in 1992 [11] is a two-dimensional coupled map model, defined on a square lattice, whose dynamical rules were inspired in a spring-block model [12] proposed to describe the dynamics of earthquakes, which is related to some empirical power-laws (like the GutenbergRitcher law). With each node of a square lattice we associate a real state variable (or 'energy') $z_{i, j}$. The model is globally driven, and each time the energy of a given site $(i, j)$ exceeds a threshold value, the system relaxes according to specific rules that will be presented in detail in section III. Within the OFC model there is a dissipation parameter $\alpha$. If $\alpha=0.25$ the dynamical variable $z_{i, j}$ of the model is conserved during the avalanche process, in the bulk of the lattice (there is always dissipation in the boundaries), but if $\alpha<0.25$ there is some dissipation also in the bulk of the system. Because of those facts, this model has been widely studied in literature. It is, at the same time, a prototype of self-organization in systems with non-conservative relaxation rules, and also a paradigm of the success of SOC ideas, since it is able to reproduce important aspects of the statistics of real earthquakes. The OFC model still attracts the attention of many researchers [13-15], because the existence of SOC in the non-conservative models is not well understood.

The random neighbor version of the OFC model (ROFC) has the same dynamical rules of the original OFC, except by the fact that it is not defined on a lattice. Now, the relaxation of any critical site affects four other sites chosen at random, instead of affecting the nearest neighbors defined by the lattice. It has been proved that the R-OFC model is critical only in the conservative regime [5-7]. In references [5] and [6] it was shown that, in the infinite-size limit, the mean value of the avalanche size, $\langle s\rangle$, is finite for all values of $\alpha<1 / q$, where $q$ is the connectivity of each site of the system. They also showed that $\langle s\rangle$ goes fast to infinity as $\alpha$ approaches $1 / q$ (the conservative limit). In reference [5], Bröker and Grassberger proposed and solved numerically an equation based on the mean value of the avalanche size and the mean energy of unstable sites 
for infinite systems. This equation has an exact solution for $\alpha=1 / q$. Their simulation results are in very good agreement with theoretical predictions. In reference [6] Chabanol and Hakim also obtained the exact solution for the equation that governs the evolution of probability distribution of energy sites, again for an infinite system. They derived a master equation, assuming that, in this limit, during an avalanche, the probability of a site relax twice is zero. Because the redistribution of energy of relaxing sites, in random models, are independent events, they may be identified with a branching process, and as a consequence, conservation is a essential ingredient to achieve criticality.

In 1996, in a controversial paper about the R-OFC model [4], Lise and Jensen developed a formalism that enabled them to perform some analytical calculations. The use of an oversimplified approximation for the energy distribution across the system (they considered a uniform distribution), in the stationary state, lead them to incorrectly predict a crossover between critical/noncritical behavior in the nonconservative regime (they concluded that, for $\alpha \geq 2 / 9$, the R-OFC model would be critical). However, although the result obtained by them is incorrect, the approach proposed in this paper, based on the analysis of the branching rate $\sigma$ is correct, clever and interesting. It has been employed with success in other works (see, for instance, [7-9,15]). Based on that approach, we were able to show that the use of a slightly better approximation for the distribution of energy in the stationary state could lead to much more reasonable results [7].

In this paper, we intend to generalize our previous analysis to deal with models with a generic coordination number (or connectivity) $q$, and address some other aspects related to the Lise-Jensen approach. Despite the fact that it had already been proved that the R-OFC model is not critical if dissipative, we think that is important to understand exactly why the ideas presented in [4] failed. We show that a better approximation for the distribution of energy is enough to lead to correct predictions. The approximation we propose for the distribution of energy in the stationary state, although still simplified if compared to the real one, also allow most of the analytical calculations proposed in [4] and can probably be useful in other situations. The paper is organized as follows: in section II, we review, with some detail, a simple model that elucidates the connection between random models with self-organized criticality and the theory of branching process (and, of course, its fundamental statistical results). We show that, for this model with $q=2$, the probability of having an avalanche of size $s$ scales with $\tau=3 / 2$, as obtained for other random conservative models with SOC. In section III, we present the dynamical rules of the OFC and R-OFC models, as well as the Lise-Jensen analytical approach. In section IV, we generalize our previous analysis to systems with any coordination number. We also discuss our results in the context of other aspects of Lise-Jensen approach, raised in a recent paper of Miller and
Boulter [15]. Finally, we present our conclusions in section $\mathrm{V}$.

\section{Self-organized criticality branching process}

The importance of the so called 'random neighbor' versions of models showing self-organized critical behavior was recognized since the introduction of this concept [16]. Because in those random models there are no spatial correlations (there is no lattice, and 'neighbors' are chosen at random), they are usually considered a kind of mean-field approximation of the corresponding lattice model. In 1995, Zapperi, Lauritsen and Stanley [17] introduced a simple model, called by them self-organized branching process (SOBP), that made clear the relation between random conservative SOC models (in the case, sandpile models) and a branching process.

A branching process [18] can be characterized by a sequence of random variables $\left\{Z_{n}\right\}_{n=0}^{\infty}, n \in \mathbf{N}$, in which $Z_{n}$ represents the total number of individuals in the $n^{\text {th }}$ generation. The number of individuals in generation $n-1$ is related to the number of individuals in the next generation $n$ through a probability $p_{i}$, that is the probability that a given individual, belonging to a given generation, gives birth to $i$ descendants, $(i=1, \ldots, q)$. This probability depends on neither what has happened in the previous generations (it is a markovian process), nor on the number of descendents that other individuals, in the same generation, eventually give birth to. Branching processes may be pictorially represented in a tree, in which $Z_{n}$ represents the number of nodes of the tree in each generation. The so called branching rate $\sigma$, defined as $\sigma=\sum_{i=0}^{\infty} i p_{i}$ corresponds to the average number of descendants a single individual gives birth to. In this context, a sequence of births can be thought as an 'avalanche', and critical branching processes, for which $\sigma=1$, are described by power laws.

Consider now the random version of the sandpile model. In the limit of an infinite system, the probability that a site topples twice in the same avalanche is zero, so it is reasonable to assume that different sites topple independently. However, because SOC models are constantly driven, the existence of open boundaries plays a fundamental role in the self-organizing process. The SOBP model was the first to take that point into account. In this model, the number of toppling sites is equivalent to the number of nodes in a branching process. A node in the tree corresponds to an active site that may generate $q$ new active sites in the next generation. Thus the size $s$ of an avalanche, usually identified with the number of sites that toppled, is equal to the total number of sites that became active throughout the generations. Boundaries were introduced in the SOBP model by allowing no more than $n$ generations for each avalanche.

In this section, we calculate, with some detail, for the 
SOBP model with $q=2$, the probability $P_{n}(s, p)$ of having an avalanche of size $s$, for any value of $p$, in a system with $n$ generations. The generating function of $Z_{n}$, in the $n$-generation [18], is defined by

$$
f_{n}(x, p)=\sum_{s=0}^{\infty} P_{n}(s, p) x^{s}
$$

in which $x$ is the expansion variable of a power series $(|x| \leq 1)$. For the SOBP model, in the $n=0$ generation, there is only one active site $(s=1)$; in generation $n=1$, there is a probability $p$ of having 2 active sites $(s=3)$ and a probability $1-p$ of having no active site $(s=1)$. Hence, we have, for $n=0$ e $n=1$, respectively,

$$
\left\{\begin{array}{l}
f_{0}(x, p)=x \\
f_{1}(x, p)=f(x, p)=(1-p) x+p x^{3}=x\left(1-p+p x^{2}\right) .
\end{array}\right.
$$

Since $f_{n+1}(x, p)=f\left[f_{n}(x, p)\right]$, for $n \geq 1$, we can write a recursion relation between $f_{n+1}(x, p)$ and $f_{n}(x, p)$ :

$$
f_{n+1}(x, p)=x\left[1-p+p f_{n}^{2}(x, p)\right] .
$$

In the limit of $n \gg 1, f_{n+1}(x, p) \simeq f_{n}(x, p)$. Solving (3) for $f(x, p)$ gives

$$
f(x, p)=\frac{1-\sqrt{1-4 x^{2} p(1-p)}}{2 x p} .
$$

Expanding (4) in power series of $x$ around $x=0$ and comparing this result with (1) leads to the following coefficients of the first terms of the series

$$
\left\{\begin{aligned}
P_{n}(1, p) & =1-p \\
P_{n}(3, p) & =p(1-p)^{2} \\
P_{n}(5, p) & =2 p^{2}(1-p)^{3} \\
& \vdots
\end{aligned}\right.
$$

For a generic $s$ we have

$$
P_{n}(s, p)=[4 p(1-p)]^{\frac{s+1}{2}} \frac{A_{s}}{2 p(s+1) !},
$$

where $A_{s}=d^{s+1} h /\left.d y^{s+1}\right|_{y=0}$, with $h(y)=\sqrt{1-y^{2}}$. It is easy to see that, for even values of $s, A_{s}=0$. For odd values of $s$ we have

$$
A_{s}=s(s-2) A_{s-2}=s \prod_{k=2}^{s-1}(s-k)^{2}=\frac{(s !)^{2}}{2^{s-1} s\left[\left(\frac{s-1}{2}\right) !\right]^{2}} .
$$

The expression $[4 p(1-p)]^{s / 2}$, presented in (6), can be re-written as

$$
[4 p(1-p)]^{s / 2}=\exp \left\{\ln [4 p(1-p)]^{s / 2}\right\}=\exp \left\{\frac{s \ln [4 p(1-p)]}{2}\right\}=\exp \left(-s / s_{c}\right),
$$

in which $s_{c}=-2 / \ln [4 p(1-p)]$. Substituting (8) into (6) gives, for the probability $P_{n}(s, p)$,

$$
P_{n}(s, p)=\sqrt{\frac{4(1-p)}{p}} \frac{\exp \left(-s / s_{c}\right) A_{s}}{2(s+1) !},
$$

where $A_{s}$ is zero if $s$ is even, and defined by (7) if $s$ is odd.

Using the Stirling relation gives

$$
\left\{\begin{array}{l}
s ! \simeq \sqrt{2 \pi} s^{(s+1 / 2)} \exp (-s) \\
(s+1) ! \simeq \sqrt{2 \pi}(s+1)^{(s+3 / 2)} \exp [-(s+1)] \simeq \sqrt{2 \pi} s^{(s+3 / 2)} \exp [-(s+1)] \\
\left(\frac{s-1}{2}\right) ! \simeq \sqrt{2 \pi}\left(\frac{s-1}{2}\right)^{s / 2} \exp [-(s-1 / 2)] \simeq \sqrt{2 \pi}\left(\frac{s}{2}\right)^{s / 2} \exp [-(s-1 / 2)]
\end{array}\right.
$$


and it is possible to write (7) as

$$
\frac{A_{s}}{2(s+1) !} \simeq \frac{1}{\sqrt{2 \pi}} s^{-3 / 2}
$$

Finally, as $s \gg 1$, expression (9) becomes

$$
P_{n}(s, p)=\sqrt{\frac{2(1-p)}{\pi p}} \frac{\exp \left(-s / s_{c}\right)}{s^{3 / 2}} .
$$

This branching process is not critical in general. However, for $p=p_{c}=1 / 2, s_{c} \rightarrow \infty$, and $\exp \left(-s / s_{c}\right) \rightarrow 1$ and the process is critical. In this case, expression (12) can be written as

$$
P_{n}\left(s, p=p_{c}\right)=\sqrt{\frac{2}{\pi}} s^{-3 / 2} .
$$

This result is exactly the probability distribution of avalanche sizes in the mean-field approximation [19, 20]. The same exponent $\tau=3 / 2$ was also obtained for other random versions of conservative models, like, for instance, the Bak-Tang-Wiesenfeld sandpile model [21] and it reflects the absence of spatial correlations. For $q \neq 2$, the procedure presented above can be generalized and the associated branching process will be critical for $p=p_{c}=1 / q$.

Also, it is quite clear from the results presented above, that we shall not expect to find criticality in non conserving models. Only in the conservative case, random selforganized critical systems are related to a branching process.

\section{The Random Olami-Feder- Christensen model}

The original OFC model [11] is a lattice model that associates to each site of the lattice a continuous state variable $E_{i, j}$, initially in the interval $\left[0, E_{c}\right)$, where $E_{c}$ is a threshold value. The system is slowly driven, and, every time the energy of a site $(i, j)$ exceeds $E_{c}$, the system relaxes. All or part of the energy of site $(i, j)$ is then distributed among its nearest neighbors. As a consequence, the energy $E$ of some of the neighbors may also exceed $E_{c}$, and the process goes on, generating an "avalanche", until $E \leq E_{c}$ again for all sites in the lattice. We assume open boundaries. The size of an avalanche is equal to the number of relaxation events. In the random version of the OFC model, every time a site becomes unstable and relaxes, "neighbors" are chosen at random.

More specifically, for the R-OFC model, the rules are:

- driving dynamics: the energy of all the sites $i, i=$ $1, \ldots, N$ is increased by $\delta E$, that is,

$$
E_{k} \rightarrow E_{k}+\delta E, \quad k=1, \ldots N
$$

- Avalanche dynamics: If any site $i$ is unstable, i. e., if its energy $E_{i} \geq E_{c}$, where $E_{c}$ is the threshold value, an avalanche is triggered and the system relaxes according to the rules:

$$
\left\{\begin{array}{l}
E_{i} \rightarrow 0 \\
E_{r n} \rightarrow E_{r n}+\alpha E_{i}
\end{array},\right.
$$

where $E_{r n}$ is the energy of $q$ sites chosen at random. The dissipation parameter $\alpha$ is defined in the range $[0,1 / q]$. If $\alpha=1 / q$ the model is conservative.

The R-OFC model, for $q=4$, was studied in the context of branching processes by Lise and Jensen [4]. They were able to calculate, after making some hypotheses and approximations, the branching rate $\sigma$ of the R-OFC model, and concluded, on the contrary of what was expected by the arguments presented in the previous section, that the model was critical in the nonconservative regime, for $\alpha \geq 2 / 9$. Their result is not correct [5,6]. We showed, however, that the problem was not in the approach used by Lise and Jensen, but in the very poor approximation they employed for the energy distribution in the stationary state [7]. We recover the intuitive approach of Lise-Jensen's paper, and used, instead, a slightly better approximation for the energy distribution. Before generalizing those results for a version of the R-OFC model with a generic coordination number, we will review the basic concepts proposed by Lise and Jensen adapting them for a general connectivity $q$.

Consider the R-OFC model during an avalanche. We will denote the energy of a generic stable site $k$ (that is, a site for which $E_{k}<E_{c}$ ) by $E_{k}^{-}$and the energy of an unstable site $\left(E_{k} \geq E_{c}\right)$ by $E_{k}^{+}$. Note that, for a site that is not toppling, $E_{k}^{-} \in\left[0, E_{c}\right]$. A generic stable site $j$ becomes unstable during an avalanche after receiving a fraction $\alpha E_{i}$ of the energy of another site $i$ that was unstable and relaxed in the previous generation. Hence, the probability that a generic site, with energy $E_{k}^{-}$, becomes unstable due to the relaxation of another site with energy $E^{+}$can be defined as the fraction of sites with energy $E$ such that $E \in\left[E_{c}-\alpha E^{+}, E_{c}\right]$ :

$$
P_{+}\left(E^{+}\right) \equiv \frac{\int_{E_{c}-\alpha E^{+}}^{E_{c}} p(E) d E}{\int_{0}^{\infty} p(E) d E},
$$

The branching ratio $\sigma$ is defined as the average number of new unstable sites generated by a single site that has just relaxed. Since the probability that an active site (a node), generates $q$ new active sites (branches) is $\left\langle P_{+}\right\rangle$, where we average over all possible values of $E^{+}\left(E^{+}>E_{c}\right)$, the branching ratio can be written as

$$
\sigma \equiv q\left\langle P_{+}\right\rangle=q \frac{\int_{E_{c}}^{\infty} P_{+}\left(E^{+}\right) p\left(E^{+}\right) d E^{+}}{\int_{E_{c}}^{\infty} p\left(E^{+}\right) d E^{+}} .
$$

The branching process is subcritical when $\sigma<1$ (the avalanches are always finite), supercritical when $\sigma \geq 1$ (the probability of having infinite avalanche is not zero and $\langle s>\rightarrow \infty)$, and critical if $\sigma=1$. Since $\sigma=q\left\langle P_{+}\right\rangle$, 
the branching process is critical when $p=\left\langle P_{+}\right\rangle=1 / q$, in agreement with what was obtained in the previous section for the SOBP model.

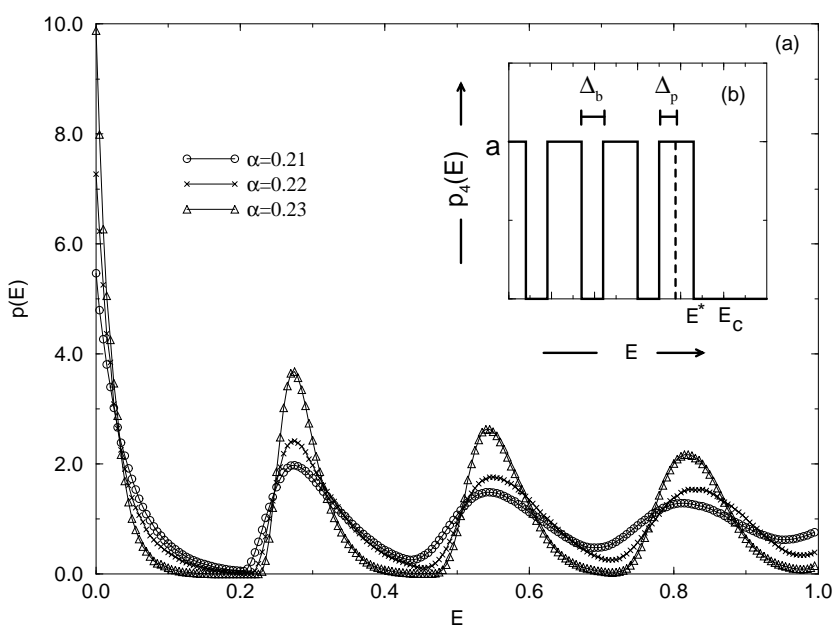

Figure 1. a) Probability distribution of energy per site $p(E)$ versus energy $E$ of R-OFC model, for $q=4$ and for different values of $\alpha: \alpha=0.21,0.22$ e 0.23 ; the width of the peaks decreases as $\alpha$ increases. b) An approximation for $p(E): p_{4}(E)$ formed by 4 peaks $(q=4)$ in which $\Delta_{p}$ is the half-width of the peaks, $\Delta_{b}$ is the width of the gaps between the peaks, and $a$ is the amplitude of peaks. We assume $E_{c} \geq E^{*}=7 \Delta_{p}+3 \Delta_{b}$.

In order to employ equation (17) to calculate the branching ratio, it is necessary to estimate the probability distribution of energy per site $p(E)$, which depends on $\alpha$. Fig. 1a, obtained numerically, exhibits $p(E)$ for the R-OFC model with $q=4$, for three different values of $\alpha$. Lise and Jensen, in their paper, approximated $p(E)$ by a uniform distribution $p_{u}(E)$ :

$$
p_{u}(E)= \begin{cases}a, & \text { for } E \in\left[0, E_{c}\right] \\ 0, & \text { for } E \in\left(E_{c}, \infty\right) .\end{cases}
$$

Hence,

$$
P_{+}^{u}\left(E^{+}\right)=\frac{\int_{E_{c}-\alpha E^{+}}^{E_{c}} p_{u}(E) d E}{\int_{0}^{E_{c}} p_{u}(E) d E}=\frac{\alpha E^{+}}{E_{c}}
$$

and

$$
\sigma^{u}=\frac{q \alpha}{E_{c}} \frac{\int_{E_{c}}^{\infty} p\left(E^{+}\right) E^{+} d E^{+}}{\int_{E_{c}}^{\infty} p\left(E^{+}\right) d E^{+}}=\frac{q \alpha\left\langle E^{+}\right\rangle}{E_{c}}
$$

where $\left\langle E^{+}\right\rangle$is the average energy of active sites defined by

$$
\left\langle E^{+}\right\rangle \equiv \frac{\int_{E_{c}}^{\infty} p\left(E^{+}\right) E^{+} d E^{+}}{\int_{E_{c}}^{\infty} p\left(E^{+}\right) d E^{+}} .
$$

Consider now a relaxing unstable site $i$, that gives the fraction $\alpha E_{i}^{+}$, of its energy to a stable site $j$, with $E_{j} \in$ $\left[E_{c}-\alpha E_{i}^{+}, E_{c}\right]$, such that, afterwards, $E_{j}^{+}=E_{j}^{-}+\alpha E_{i}^{+}$. Assuming that $\left\langle E_{j}^{+}\right\rangle=\left\langle E_{i}^{+}\right\rangle=\left\langle E^{+}\right\rangle$(what is reasonable in the stationary state and was another assumption made by Lise and Jensen) gives

$$
\left\langle E^{+}\right\rangle=\frac{\left\langle E^{-}\right\rangle}{1-\alpha},
$$

where $\left\langle E^{-}\right\rangle$is the average value of the energy of all stable sites that may become unstable, and can be written as

$$
\left\langle E^{-}\right\rangle \equiv \frac{\int_{E_{c}-\alpha E^{+}}^{E_{c}} E^{-} p\left(E^{-}\right) d E^{-}}{\int_{E_{c}-\alpha E^{+}}^{E_{c}} p\left(E^{-}\right) d E^{-}} \approx \frac{\int_{E_{c}-\alpha\left\langle E^{+}\right\rangle}^{E_{c}} E^{-} p\left(E^{-}\right) d E^{-}}{\int_{E_{c}-\alpha\left\langle E^{+}\right\rangle}^{E_{c}} p\left(E^{-}\right) d E^{-}} .
$$

The assumption that $p(E)$ is uniform leads to

$$
\left\langle E^{-}\right\rangle^{u}=\frac{E_{c}^{2}-\left(E_{c}-\alpha\left\langle E^{+}\right\rangle\right)^{2}}{2 \alpha\left\langle E^{+}\right\rangle}=E_{c}-\frac{\alpha\left\langle E^{+}\right\rangle}{2}
$$


and to

$$
\left\langle E^{+}\right\rangle^{u}=\frac{2 E_{c}}{2-\alpha} .
$$

Finally, substituting (25) into (20), we get, for the branching rate

$$
\sigma^{u}=\frac{2 q \alpha}{2-\alpha} .
$$

The process is critical if $\sigma=1$, what happens if

$$
\alpha=\alpha_{c}=\frac{2}{2 q+1} .
$$

For $q=4$, Lise-Jensen's result is recovered, that is $\alpha_{c}=2 / 9$.

\section{A better approximation for the en- ergy distribution $p(E)$}

Suppose that we now assume a slightly more realistic approximation for $p(E)$,

$$
p_{q}(E)= \begin{cases}a, & \text { for } E \in I_{i}, i=1, \ldots, q \\ 0, & \text { for other cases }\end{cases}
$$

where $I_{1}=\left[0, \Delta_{p}\right]$ and $I_{i}=\left[(2 i-3) \Delta_{p}+(i-1) \Delta_{b},(2 i-\right.$ 1) $\left.\Delta_{p}+(i-1) \Delta_{b}\right], i=2, \ldots, q \cdot p(E)$ now is a distribution characterized by $q$ 'square peaks' of width $2 \Delta_{p}$ (see figure $1 \mathrm{~b}$ for $q=4) . \Delta_{b}$ is the width of the gaps between the peaks. Note that $E^{*}=(q-1) \Delta_{b}+(2 q-1) \Delta_{p}$ is the maximum value of $E$ for which $p_{q}(E) \neq 0$, in other words $E_{c} \geq E^{*}$.

If $\Delta_{b} \rightarrow 0$ and $\Delta_{p} \rightarrow E_{c} /(2 q-1)$, the uniform approximation is recovered, since $p_{q}(E)=p_{u}(E)$. On the other hand, if $\Delta_{p} \rightarrow 0$ and $\Delta_{b} \rightarrow \alpha E_{c}, p_{q}(E)$ tends to $q$ delta functions, that is what we would obtain in the conservative case (see reference [5]).

We repeat the same steps exhibited in previous subsection, using now the distribution (28):

$$
P_{+}\left(E^{+}\right)=\frac{\int_{E_{c}-\alpha E^{+}}^{E_{c}} p_{q}(E) d E}{\int_{0}^{\infty} p_{q}(E) d E}=\frac{\int_{E_{c}-\alpha E^{+}}^{E_{c}} p_{q}(E) d E}{(2 q-1) a \Delta_{p}},
$$

where the inferior limit $E_{c}-\alpha E^{+}$belongs to any of the intervals $I_{i}, i=1, \ldots, q$, the denominator is

$$
\begin{aligned}
\int_{0}^{\infty} p_{q}(E) d E & =\int_{0}^{\Delta_{p}} a d E+\sum_{i=2}^{q}\left[\int_{(2 i-3) \Delta_{p}+(i-1) \Delta_{b}}^{(2 i-1) \Delta_{p}+(i-1) \Delta_{b}} a d E\right]= \\
& =a \Delta_{p}+(q-1)(2 a) \Delta_{p}=(2 q-1) a \Delta_{p}
\end{aligned}
$$

and the numerator is

$$
\begin{aligned}
\int_{E_{c}-\alpha E^{+}}^{E_{c}} p_{q}(E) d E & =\int_{E_{c}-\alpha E^{+}}^{(2 i-1) \Delta_{p}+(i-1) \Delta_{b}} a d E+(q-i) 2 a \Delta_{p}= \\
& =a\left[(2 q-1) \Delta_{p}+(i-1) \Delta_{b}-\left(E_{c}-\alpha E^{+}\right)^{i}\right] .
\end{aligned}
$$

The superscript $i$ indicates in which of the intervals $I_{i}, i=1, \ldots, q$, the expression $\left(E_{c}-\alpha E^{+}\right)$is located. Putting (30) and (31) into (29), leads to

$$
P_{+}^{i}\left(E^{+}\right)=1+\frac{(i-1) \Delta_{b}}{(2 q-1) \Delta_{p}}-\frac{E_{c}}{(2 q-1) \Delta_{p}}+\frac{\alpha E^{+}}{(2 q-1) \Delta_{p}},
$$

and, for the branching ratio (17)

$$
\sigma^{i}=q\left\langle P_{+}\right\rangle^{i}=q\left[1+\frac{(i-1) \Delta_{b}}{(2 q-1) \Delta_{p}}-\frac{E_{c}}{(2 q-1) \Delta_{p}}+\frac{\alpha\left\langle E^{+}\right\rangle^{i}}{(2 q-1) \Delta_{p}}\right],
$$

where $\left\langle P_{+}\right\rangle^{i}$ means the average over all possible values of $E^{+}$such that $E_{c}-\alpha E^{+} \in I_{i}$. 
Analogously to (22), we also have

$$
\left\langle E^{+}\right\rangle^{i}=\frac{\left\langle E^{-}\right\rangle^{i}}{1-\alpha}
$$

where $\left\langle E^{+}\right\rangle^{i}$ and $\left\langle E^{-}\right\rangle^{i}$ are, respectively, the average values of energy of unstable and stable sites. From (28) and (23) we get

$$
\left\langle E^{-}\right\rangle^{i}=\frac{(2 q-1)^{2} \Delta_{p}^{2}+(i-1)^{2} \Delta_{b}^{2}+x_{i} \Delta_{p} \Delta_{b}-\left[E_{c}-\alpha\left\langle E^{+}\right\rangle^{i}\right]^{2}}{2\left[(2 q-1) \Delta_{p}+(i-1) \Delta_{b}-E_{c}+\alpha\left\langle E^{+}\right\rangle^{i}\right]},
$$

in which

$$
\begin{aligned}
x_{i} & =2\left[(2 i-1)(i-1)+\sum_{j=i}^{q-1} 2 j\right]= \\
& =2[(2 i-1)(i-1)+(q-1) q-i(i-1)]= \\
& =2\left[(i-1)^{2}+(q-1) q\right],
\end{aligned}
$$

with $i=1, \ldots, q$. Substituting (35) into equation (34), we obtain a second-order equation whose solution is

$$
\left\langle E^{+}\right\rangle^{i}=\frac{E_{c}}{\alpha(2-\alpha)}-\frac{\left[(2 q-1) \Delta_{p}+(i-1) \Delta_{b}\right](1-\alpha)}{\alpha(2-\alpha)} \pm \frac{\sqrt{y_{i}}}{2 \alpha(2-\alpha)},
$$

with

$$
y_{i}=4\left\{E_{c}(1-\alpha)-\left[(2 q-1) \Delta_{p}+(i-1) \Delta_{b}\right]\right\}^{2}+8 \alpha(2-\alpha)[(i-1)(i-2 q)+q(q-1)] \Delta_{p} \Delta_{b} .
$$

Substituting (37) into (33) and imposing that $\sigma=1$ (critical condition), we get

$$
-2 q E_{c}\left(1-\alpha_{c}\right)+2(2 q-1)\left(q-2+\alpha_{c}\right) \Delta_{p}+2 q(i-1) \Delta_{b} \pm q \sqrt{y_{i}}=0,
$$

where $y_{i}$ is given by the expression (38). It is possible to show, from (39), that

$$
A_{i} \alpha_{c}^{2}+B_{i} \alpha_{c}+C_{i}=0
$$

where the constants $A_{i}, B_{i}$ and $C_{i}$ are given by

$$
\left\{\begin{aligned}
A_{i}= & 2 q(2 q-1) E_{c}+(2 q-1)^{2} \Delta_{p}+2 q^{2}[(i-1)(i-2 q)+q(q-1)] \Delta_{b} \\
B_{i}= & -6 q(2 q-1) E_{c}+2(q-2)(2 q-1)^{2} \Delta_{p}+ \\
& +2 q\left\{(i-1)[(2 q-1)-2 q(i-2 q)]-2 q^{2}(q-1)\right\} \Delta_{b} \\
C_{i}= & 4(2 q-1)\left[q E_{c}+(1-q)(2 q-1) \Delta_{p}-q(i-1) \Delta_{b}\right]
\end{aligned}\right.
$$

The polynomial (40) can be written as

$$
A_{i} \alpha_{c}^{2}+B_{i} \alpha_{c}+C_{i}=\left(\alpha_{c}-2\right)\left[A_{i} \alpha_{c}+\left(2 A_{i}+B_{i}\right)\right]+R_{i},
$$

where $R_{i}=4 A_{i}+2 B_{i}+C_{i}=0$. The two solutions of (42) are $\alpha_{c}=2$ and $\alpha_{c}=-\left(2 A_{i}+B_{i}\right) / A_{i}$. Since, in our case, $\alpha \in[0,1 / q]$, only the second solution is valid.

The uniform distribution of energy, $p_{u}(E)$, is recovered if $\Delta_{p} \rightarrow E_{c} /(2 q-1)$ and $\Delta_{b} \rightarrow 0$. In this case the coefficients expressed by (41) become

$$
\left\{\begin{array}{l}
A_{i}=A=(2 q+1)(2 q-1) E_{c} \\
B_{i}=B=-4(q+1)(2 q-1) E_{c} \\
C_{i}=C=4(2 q-1) E_{c}
\end{array} .\right.
$$

and $\alpha_{c}=2 /(2 q+1)$, which is equal to (27); as expected, in the particular case of $q=4, \alpha_{c}=2 / 9$.

Let now consider the other limit. If $\Delta_{p} \rightarrow 0$ and $\Delta_{b} \rightarrow \alpha_{c} E_{c}$ the energy distribution becomes a sequence of delta functions, and that is exactly what is expected for the conservative R-OFC model. In this case the coefficients (41) become

$$
\left\{\begin{array}{l}
A_{i}=q E_{c}\left\{2(2 q-1)+2 q \alpha_{c}[(i-1)(i-2 q)+q(q-1)]\right\} \\
B_{i}=2 q E_{c}\left\{-3(2 q-1)+\left[(i-1)(2 q-1)-2 q(i-1)(i-2 q)-2 q^{2}(q-1)\right] \alpha_{c}\right\} \\
C_{i}=4 q(2 q-1) E_{c}\left[1-(i-1) \alpha_{c}\right]
\end{array} .\right.
$$


and equation (40) becomes

$$
a_{3} \alpha_{c}^{3}+a_{2} \alpha_{c}^{2}+a_{1} \alpha_{c}+a_{0}=0
$$

with

$$
\left\{\begin{array}{l}
\left.a_{3}=2 q[(i-1)(i-2 q)+q(q-1))\right] \\
a_{2}=2\left[(2 q-1) i-2 q(i-1)(i-2 q)-2 q^{2}(q-1)\right] \\
a_{1}=-2(2 q-1)(1+2 i) \\
a_{0}=4(2 q-1)
\end{array} .\right.
$$

Dividing (45) by $\left(\alpha_{c}-2\right)$ yields

$$
b_{2} \alpha_{c}^{2}+b_{1} \alpha_{c}+b_{0}=0
$$

where

$$
\left\{\begin{array}{l}
b_{2}=2 q[(i-1)(i-2 q)+q(q-1)] \\
b_{1}=2 i(2 q-1) \\
b_{0}=-2(2 q-1)
\end{array} .\right.
$$

If $i=q$, we have $b_{2}=0$, and $\alpha_{c}=1 / q$. If $i<q$, it is not hard to prove (see appendix) that the solutions of equation (47) are such that $\alpha_{c}>1 / q$ or $\alpha_{c}<0$, what is impossible. We conclude then that, in the conservative limit, we must have $i=q$ and $\alpha_{c}=1 / q$ (for $q=4, \alpha_{c}=1 / 4$ ) as expected.

In the generic case $\Delta_{p} \geq 0, \Delta_{b} \geq 0$ with the restriction that $E^{*} \geq E_{c}$, that is,

$$
\frac{E^{*}}{E_{c}}=(2 q-1) \gamma_{p}+(q-1) \gamma_{b} \leq 1
$$

where $\gamma_{p}=\Delta_{p} / E_{c}$ and $\gamma_{b}=\Delta_{b} / E_{c}$. The question we now ask is: Are there any values of $\alpha<1 / q$ for which $\sigma$ may be equal to 1 ? In other words, if we approximate $p(E)$ by the distribution of energy given in (28), can the model still display a critical behavior in the nonconservative case?

In this case, the second solution of (45) may be written as

$$
\alpha_{c}=\frac{P}{Q}
$$

where

$$
P=1-\frac{(2 q-1)(q-1)}{q} \gamma_{p}-(i-1) \gamma_{b}
$$

and

$$
Q=1+\frac{(2 q-1)}{2 q} \gamma_{p}+\frac{q[(i-1)(i-2 q)+q(q-1)]}{2 q-1} \gamma_{b},
$$

for all $q>0, \gamma_{p} \geq 0, \gamma_{b} \geq 0$.

Let us consider the regions of the parameter space $\gamma_{p} \times$ $\gamma_{b}, \forall I_{i}$, for which $0<\alpha_{c} \leq 1 / q$, under the restriction (49). In order to have $\alpha_{c}>0$, (51) must be positive, since $Q>0$, $\forall i$. This is guaranteed by inequality (49), $\forall \gamma_{p} \geq 0$ and $\gamma_{b} \geq 0$, because

$$
1 \geq(2 q-1) \gamma_{p}+(q-1) \gamma_{b}>\frac{(2 q-1)(q-1)}{q} \gamma_{p}+(i-1) \gamma_{b}
$$

Supposing now that $\alpha_{c} \leq 1 / q$, (50) leads to the following inequality:

$$
1-\frac{(2 q-1)\left(2 q^{2}-2 q+1\right)}{2 q(q-1)} \gamma_{p}-\frac{q\left[(i-1)^{2}+q(q-1)\right]}{(q-1)(2 q-1)} \gamma_{b} \leq 0
$$

that, for the particular case of $q=4$, takes the form

$$
1-\frac{175}{24} \gamma_{p}-\frac{4\left[(i-1)^{2}+12\right]}{21} \gamma_{b} \leq 0 .
$$

This particular case was presented in Fig. 2, for each of the intervals $I_{i}$, just to illustrate the general discussion we will present below. In this figure, the shaded regions, for each of the 4 peaks of $p_{4}(E)$, indicate pairs of values of $\left(\gamma_{p}, \gamma_{b}\right)$ compatible with the conditions imposed by inequalities (49) and (55). They represents the values of $\left(\Delta_{p}, \Delta_{b}\right)$ for which we cannot guarantee (based on logical arguments and restrictions as the ones deduced above) that the model is not critical. From now on we will call them "critical regions" in the parameter space. For all values of $i$ we observe that there is a small shaded region, which shows us that there is a non-zero, but small, probability of having criticality, in the physically accessible range of the parameter $\alpha$. That probability decreases as $i$ increases, since the probability that a stable site becomes unstable is higher for larger value of $i$.

From figures $2 \mathrm{a}$ and $2 \mathrm{~b}$, we see that the values of $\gamma_{b}$ associated with the shaded area (the values of $\gamma_{p}$ and $\gamma_{p}$ for which the model is possibly critical) are very small. $\gamma_{b}$ is related to the size of the gaps between the peaks. That means that, to allow criticality, $p_{4}(E) \sim p_{u}(E)$ and very far from the expected $p(E)$ for $\alpha \approx 0.25$. In figures $2 \mathrm{c}$ and $2 \mathrm{~d}$, the values of $\gamma_{b}$ are a bit larger, but still not compatible with the range of values of those parameters that would approximate the four peak distribution to the 'real' distribution shown in figure 1a (where $\gamma_{p} \simeq 0.08$ e $\gamma_{b} \simeq 0.1$ ). 

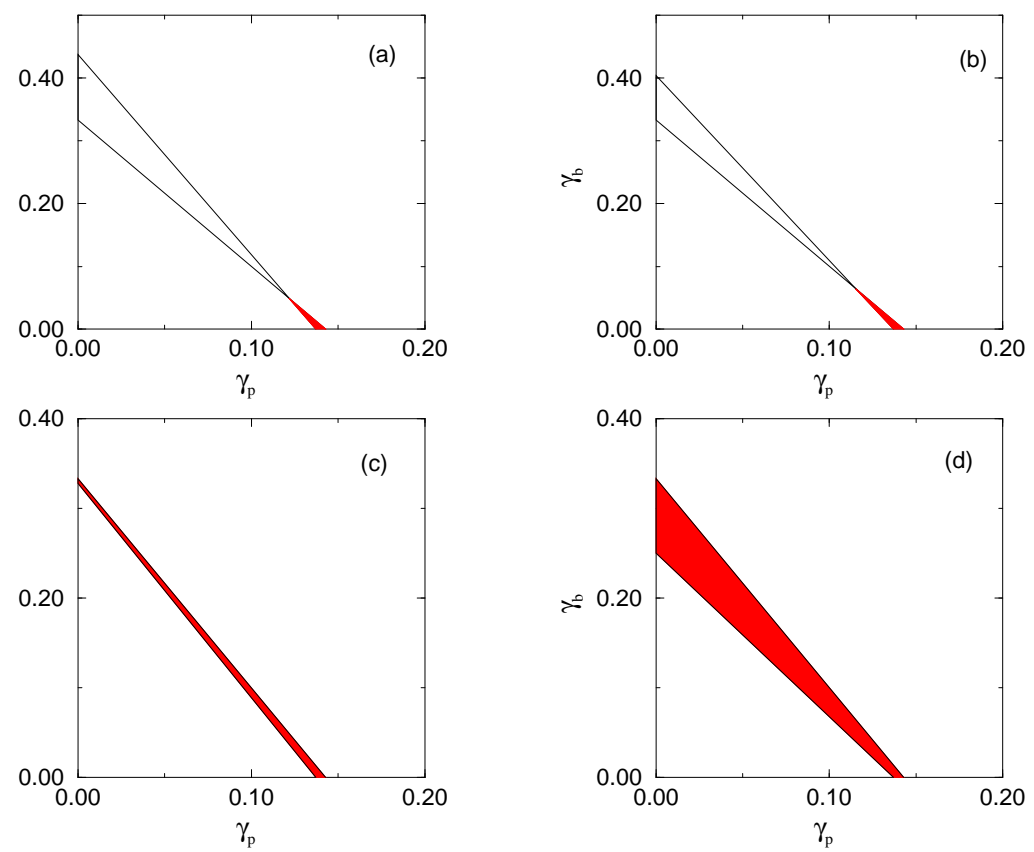

Figure 2. The parameter space of $p_{q}(E)$, with $q=4$ in terms of $\gamma_{p}=\Delta_{p} / E_{c}$ and $\gamma_{b}=\Delta_{b} / E_{c}$. The shaded regions correspond to limited regions by the interception between $\alpha \leq 1 / 4$ and $7 \gamma_{p}+3 \gamma_{b} \leq 1$. Depending on the value of $E_{c}-\alpha E^{+}$, there are 4 ( $q=4$ ) cases: (a) $E_{c}-\alpha E^{+} \epsilon\left[0, \Delta_{p}\right]$; (b) $E_{c}-\alpha E^{+} \epsilon\left[\Delta_{p}+\Delta_{b}, 3 \Delta_{p}+\Delta_{b}\right]$; (c) $E_{c}-\alpha E^{+} \epsilon\left[3 \Delta_{p}+2 \Delta_{b}, 5 \Delta_{p}+2 \Delta_{b}\right]$; e (d) $E_{c}-\alpha E^{+} \epsilon$ $\left[5 \Delta_{p}+3 \Delta_{b}, 7 \Delta_{p}+3 \Delta_{b}\right]$.

In the case of a generic $q$ we can state that:

a) there is no value of $q$ such that the lines, that limit the critical regions, coincide, since the coefficients of $\gamma_{b}$, for the lines corresponding to inequalities (49) and (54), never have identical values;

b) for any $i \leq q$, there is always a region, in the parameter space, for which the model may be critical. This can be proved with the same argument employed in item a).

c) the smaller the value of $i$, the smaller the region, in the parameter space, for which the model may be critical; for the highest value of $i, i=q$, the shaded area $A_{q}$ decreases with $q$ according to a power law given by

$$
\begin{aligned}
A_{q} & =\frac{1}{2(q-1)(2 q-1)}-\frac{2(q-1)}{(2 q-1)\left(2 q^{2}-2 q+1\right)}= \\
& =\frac{1}{2(q-1)\left(2 q^{2}-2 q+1\right)} .
\end{aligned}
$$

Moreover,

$$
\lim _{q \rightarrow \infty} A_{q}=\lim _{q \rightarrow \infty} \frac{1}{2(q-1)\left(2 q^{2}-2 q+1\right)}=0 .
$$

d) For any $q>2$, the lines that define the region, in the parameter space, where we cannot exclude the critical behavior, intercept each other for $i<q-1$.

In order to see that, we compare inequalities (49) and (54). The lines intercept when

$$
\frac{(2 q-1)(q-1)}{q\left[(i-1)^{2}+q(q-1)\right]}>\frac{1}{q-1} .
$$

This expression can be re-written in the following form:

$$
q i^{2}-2 q i-q^{3}+4 q^{2}-3 q+1<0 .
$$

Solving this equation for $i$ gives

$$
i_{ \pm}=1 \pm \frac{\sqrt{D}}{2 q}
$$

where $D=4 q\left(q^{3}-4 q^{2}+4 q-1\right)$. For $q=1$ we have $D=0$, so $i=q=1$; for $q=2$, it follows that $D<0$, so there is no value of $i$ such that the lines intercept each other. For $q>2$, we see that $D>0$, what means that there is some value of $i$ such that the lines intercept each other. $i_{-}$ is always smaller than 1 . In this case, it is easy to prove, by contradiction, that $i_{+}<q-1$.

Recently, Miller and Boulter [15] used this branchingrate approach in a more general discussion about the criticality of the OFC model in the non-conservative regime. 
In order to argue that the OFC model indeed organizes itself towards criticality (regardless of whether it is critical or not), they calculated the branching rate and compared the behavior of both OFC and R-OFC models. They revisited Lise-Jensen's approach, but now without assuming that

$$
\left\langle E_{j}^{+}\right\rangle=\left\langle E_{i}^{+}\right\rangle=\left\langle E^{+}\right\rangle .
$$

Instead, they estimated a recursion relation connecting the average energy of active sites between two successive generations in an avalanche, at the beginning of the organization process. With this approach they were able to get, for the ROFC model, better fittings for the average energy of active sites, $\left\langle E^{+}\right\rangle$and, as a consequence, obtained also better results for the branching rate $\sigma$, versus the dissipation parameter $\alpha$ (than the one obtained with Lise-Jensen approximation (61)).

We think that, even if we assume (61), what is reasonable for the stationary state, the use of a better approximation for the energy distribution $p(E)$, like the one suggested by us (see (28)), will also led to better fittings for both $\left\langle E^{+}\right\rangle$ and $\sigma$ versus $\alpha$. In the particular case of $i=q-$ when the probability of having criticality is greatest (see figure $2 \mathrm{~d}$ ) the expression (38) becomes

$$
y_{i=q}=2\left|E_{c}(1-\alpha)-\left[(2 q-1) \Delta_{p}+(i-1) \Delta_{b}\right]^{2}\right|,
$$

and the solutions (37) can be rewritten as

$$
\begin{aligned}
& \left\langle E^{+}\right\rangle_{(+)}^{q}=\left\{\begin{array}{cc}
\left(E_{c}-E^{*}\right) / \alpha, & \text { if } \alpha<1-\left(E_{c} / E^{*}\right) \\
\left(E_{c}+E^{*}\right) /(2-\alpha), & \text { if } \alpha>1-\left(E_{c} / E^{*}\right)
\end{array},\right. \\
& \left\langle E^{+}\right\rangle_{(-)}^{q}=\left\{\begin{array}{cc}
\left(E_{c}+E^{*}\right) /(2-\alpha), & \text { if } \alpha<1-\left(E_{c} / E^{*}\right) \\
\left(E_{c}-E^{*}\right) / \alpha, & \text { if } \alpha>1-\left(E_{c} / E^{*}\right)
\end{array},\right.
\end{aligned}
$$

where $E^{*}=(2 q-1) \Delta_{p}+(q-1) \Delta_{q}$.

Analogously, the expressions for (33) become

$$
\begin{aligned}
& \sigma_{(+)}^{q}=\left\{\begin{array}{cc}
0, & \text { if } \alpha<1-\left(E_{c} / E^{*}\right) \\
2 q\left[E^{*}-E_{c}(1-\alpha)\right] / \Delta_{p}(2 q-1) \alpha(2-\alpha), & \text { if } \alpha>1-\left(E_{c} / E^{*}\right)
\end{array},\right. \\
& \sigma_{(-)}^{q}=\left\{\begin{array}{cc}
2 q\left[E^{*}-E_{c}(1-\alpha)\right] / \Delta_{p}(2 q-1) \alpha(2-\alpha), & \text { if } \alpha<1-\left(E_{c} / E^{*}\right) \\
0, & \text { if } \alpha>1-\left(E_{c} / E^{*}\right)
\end{array} .\right.
\end{aligned}
$$

Examining theses expressions we notice that they also do not increase linearly with $\alpha$, as in what was called "LiseJensen approximation" in [15]. We think that this is an evidence that, if we define $\sigma$ as a function of $\sigma^{i}$, we also would obtain, for $\sigma \times \alpha$, a curve much closer to the simulation results obtained by Miller and Boulter [15].

\section{Conclusions}

In this paper, we discuss the importance, to the analysis of random versions of models with self-organized criticality, of the the branching rate approach introduced in ([4]). We review the branching rate approach and we calculate, in detail, the exponent $\tau$ that characterizes the probability distribution of avalanche sizes. The result obtained, $\tau=3 / 2$, corroborates the connection between random SOC models and branching processes.

We concentrate our attention in the R-OFC model, gen- eralizing our previous results to systems with an arbitrary connectivity $q$. Our calculations made clear how sensitive the results, to the approximation made for the energy distribution, necessary to calculate the branching rate $\sigma$.

We were also able to show that, for the more realistic approximation to the energy distribution $p_{q}(E)$ in the stationary state $(p(E)$ is approximated by $q$ square peaks) there is only a small region of parameter space, $\Delta_{p} \times \Delta_{b}$, for which we cannot exclude criticality in the non conservative case. However, the values of $\gamma_{p}$ and $\gamma_{b}$ for which our simplified $p_{q}(E)$ is close to the distribution obtained from numerical simulations do not belong to those regions.

We suggest that this better, but still simple approximation, might also be able to reproduce the numerical simulations of the average energy of active sites and the branching rate for R-OFC model as functions of the dissipation parameter $\alpha$. 


\section{Appendix}

In that appendix we prove that if $i<q$, the solutions of (47) are such that $\alpha_{c}>1 / q$ or $\alpha_{c}<0$. In other words, we must assume $i=q$ in order to have $\alpha_{c} \in[0,1 / q]$.
Dem] The solutions of (47) are given by

$$
\alpha_{c}=\frac{-2 i(2 q-1) \pm \sqrt{D}}{4 q[(i-1)(i-2 q)+q(q-1)]},
$$

with $D=4 i^{2}(2 q-1)^{2}+16 q(2 q-1)[(i-1)(i-2 q)+$ $q(q-1)]$. Then, under the assumption $i<q$, and setting

$$
\begin{aligned}
(i-1)(i-2 q)+q(q-1) & =i^{2}-2 q i-i+2 q+q^{2}-q= \\
& =(q-i)^{2}+(q-i)>0 .
\end{aligned}
$$

we get $\sqrt{D}>2 i(2 q-1)$. This relation guarantees that, if one solution is negative, the other is positive.

Suppose now that the positive solution, $\alpha_{c}>0$, is such that $\alpha_{c} \leq 1 / q$; so $\alpha_{c}^{2} \leq 1 / q^{2}$. Then the inequality can be written as follows:

$$
b_{2} \alpha_{c}^{2}+b_{1} \alpha_{c}+b_{0} \leq \frac{b_{2}}{q^{2}}+\frac{b_{1}}{q}+b_{0} .
$$

Using equation (47), (69) is then

$$
b_{2}+q b_{1}+q^{2} b_{0} \geq 0 \text {. }
$$

Putting the coefficients (48) into (70) leads to

$$
\begin{aligned}
b_{2}+q b_{1}+q^{2} b_{0} & =2 q\left[(i-1)^{2}+q(q-1)-(2 q-1)(i-1)+i(2 q-1)-q(2 q-1)\right]= \\
& =2 q\left[(i-1)^{2}+q(q-1)-(2 q-1)(q-1)\right]= \\
& =2 q\left[(i-1)^{2}-(q-1)^{2}\right] \geq 0
\end{aligned}
$$

It is easy to see that, when $i<q$, we have a contradiction. Therefore, for $i<q, \alpha_{c}<0$ or $\alpha_{c}>1 / q$.

\section{References}

[1] P. Bak, C. Tang, and K. Wiesenfeld, Phys. Rev. Lett. 59, 381 (1987); P. Bak, C. Tang, and K. Wiesenfeld, Phys. Rev. A 38 , 364 (1988).

[2] D. Dhar and R. Ramaswamy, Phys. Rev. Lett. 63, 1659 (1989); D. Dhar, Phys. Rev. Lett. 64, 1613 (1990).

[3] A. Vespignani and S. Zapperi, Phys. Rev. Lett. 78, 4793 (1997); A. Vespignani and S. Zapperi, Phys. Rev. E 57, 6345 (1998).

[4] S. Lise and H. J. Jensen, Phys. Rev. Lett. 76, 2326 (1996).

[5] H-M. Bröker and P. Grassberger, Phys. Rev. E 56, 3944 (1997).

[6] M-L. Chabanol and V. Hakim, Phys. Rev. E 56, R2343 (1997).

[7] S. T. R. Pinho, C. P. C. Prado, and O. Kinouchi, Physica A 257, 488 (1998).

[8] O. Kinouchi, S. T. R. Pinho, and C. P. C. Prado, Phys. Rev. E 58, 3997 (1998).

[9] O. Kinouchi and C. P. C. Prado, Phys. Rev. E 59, 4964 (1999).

[10] L. Pietronero, A. Vespignani, and S. Zapperi, Phys. Rev. Lett. 72, 1690 (1994).
[11] Z. Olami, H. J. S. Feder, and K. Christensen, Phys. Rev. Lett. 68, 1244 (1992).

[12] R. Burridge and L. Knopoff, Bull. Seimol. Soc. Am. 57, 341 (1967).

[13] J. X. de Carvalho and C. P. C. Prado, Phys. Rev. Lett. 84, 4006 (2000).

[14] S. Lise and M. Paczuski, Phys. Rev. E 63, 036111 (2001).

[15] G. Miller and C. J. Boulter, Phys. Rev. E 66, 016123 (2002).

[16] K. Christensen, Self-organization in models of sandpiles, earthquakes and flashing fireflies, $\mathrm{PhD}$ Thesis, (University of Aarhus, Denmark, 1992).

[17] S. Zapperi, K. B. Lauritsen, and H. E. Stanley, Phys. Rev. Lett. 75, 4071 (1995).

[18] T. E. Harris, The Theory of Branching Processes, (Dover Publications, New York, 1989).

[19] D. Dhar and S. N. Majumdar, J. Phys. A 23, 4333 (1990).

[20] S. A. Janowsky and C. A. Laberge, J. Phys. A 26, L973 (1993).

[21] K. Christensen and Z. Olami, Phys. Rev. E 48, 3361 (1993). 